\title{
Circulating Biomarkers and Cardiac Structure and Function in Rheumatoid Arthritis
}

\begin{abstract}
Masatake Kobayashi ${ }^{1+}$, Maria Betânia Ferreira ${ }^{2,3,4 t}$, Rita Quelhas Costa ${ }^{5}$, Tomás Fonseca ${ }^{6}$, José Carlos Oliveira ${ }^{7}$, António Marinho ${ }^{3,6}$, Henrique Cyrne Carvalho ${ }^{2,3,8}$, Nicolas Girerd $^{1}$, Patrick Rossignol ${ }^{1}$, Faiez Zannad ${ }^{1}$, Patrícia Rodrigues ${ }^{2,3,8}$ and João Pedro Ferreira ${ }^{1,9 *}$

${ }^{1}$ Université de Lorraine, INSERM, Centre d'Investigations Cliniques Plurithématique 1433, INSERM U1116, CHRU de Nancy and F-CRIN INI-CRCT, Nancy, France, ${ }^{2}$ Unit of Multidisciplinary Research in Biomedicine, Porto, Portugal, ${ }^{3}$ Instituto de Ciências Biomédicas Abel Salazar, School of Medicine and Biomedical Sciences, University of Porto, Porto, Portugal, ${ }^{4}$ Hospital da Luz Arrábida, Porto, Portugal, ${ }^{5}$ Internal Medicine Department, Centro Hospitalar de Entre o Douro e Vouga, Aveiro, Portugal, ${ }^{6}$ Internal Medicine Department, Centro Hospitalar Universitário Do Porto, Porto, Portugal, ${ }^{7}$ Clinical Chemistry Service, Centro Hospitalar Universitário Do Porto, Porto, Portugal, ${ }^{8}$ Cardiology Department, Centro Hospitalar Universitário Do Porto, Porto, Portugal, ${ }^{9}$ Department of Surgery and Physiology, Cardiovascular Research and Development Center, Faculty of Medicine of the University of Porto, Porto, Portugal
\end{abstract}

OPEN ACCESS

Edited by:

Sophie Mavrogeni,

Onassis Cardiac Surgery

Center, Greece

Reviewed by:

Attila Kovacs,

Semme/weis University, Hungary

Maria Aurora Morales,

National Research Council (CNR), Italy

*Correspondence:

João Pedro Ferreira

j.ferreira@chru-nancy.fr

†These authors share first authorship

Specialty section:

This article was submitted to

Cardiovascular Imaging

a section of the journal

Frontiers in Cardiovascular Medicine

Received: 07 August 2021

Accepted: 13 October 2021

Published: 18 November 2021

Citation:

Kobayashi M, Ferreira MB, Costa RQ,

Fonseca T, Oliveira JC, Marinho A,

Carvalho HC, Girerd N, Rossignol P,

Zannad $F$, Rodrigues $P$ and

Ferreira JP (2021) Circulating

Biomarkers and Cardiac Structure and Function in Rheumatoid Arthritis.

Front. Cardiovasc. Med. 8:754784.

doi: $10.3389 /$ fcrm.2021.754784
Background: Rheumatoid arthritis $(\mathrm{RA})$ increases the risk for abnormalities of the cardiac structure and function, which may lead to heart failure (HF). Studying the association between circulating biomarkers and echocardiographic parameters is important to screen patients with RA with a higher risk of cardiac dysfunction.

Aim: To study the association between circulating biomarkers and echocardiographic parameters in patients with RA.

Methods: Echocardiography was performed in 355 patients with RA from RA Porto cohort and the associations between echocardiographic characteristics and 94 circulating biomarkers were assessed. These associations were also assessed in the Metabolic Road to Diastolic Heart Failure (MEDIA-DHF) [392 patients with HF with preserved ejection fraction (HFpEF)] and the Suivi Temporaire Annuel Non-Invasif de la Santé des Lorrains Assurés Sociaux (STANISLAS) (1,672 healthy population) cohorts.

Results: In the RA Porto cohort, mean age was $58 \pm 13$ years, 23\% were males and mean RA duration was $12 \pm 10$ years. After adjustment and multiple testing correction, left ventricular mass index (LVMi), left atrial volume index (LAVi), and $E / e^{\prime}$ were independently associated with biomarkers reflecting inflammation [i.e., bone morphogenetic protein 9 (BMP9), pentraxin-related protein 3 (PTX3), tumor necrosis factor receptor superfamily member 11a (TNFRSF11A)], extracellular matrix remodeling [i.e., placental growth factor (PGF)], congestion [i.e., N-terminal pro-brain natriuretic peptide (NT-proBNP), adrenomedullin (ADM)], and myocardial injury (e.g., troponin). Greater LVMi [hazard ratio (HR) $(95 \% \mathrm{Cl})$ per $1 \mathrm{~g} / \mathrm{m}^{2}=1.03$ (1.02-1.04), $p<0.001]$, LAVi [HR $(95 \% \mathrm{Cl})$ per $1 \mathrm{ml} / \mathrm{m}^{2}=1.03(1.01-1.06)$, $p<0.001]$, and $\mathrm{E} / \mathrm{e}^{\prime}[\mathrm{HR}(95 \% \mathrm{Cl})$ per $1=1.08$ (1.04-1.13), $p<0.001]$ were associated with higher rates of cardiovascular events. These associations were externally replicated in patients with HFpEF and asymptomatic individuals. 
Conclusion: Circulating biomarkers reflecting inflammation, extracellular matrix remodeling, congestion, and myocardial injury were associated with underlying alterations of cardiac structure and function. Biomarkers might be used for the screening of cardiac alterations in patients with RA.

Keywords: rheumatoid arthritis, heart failure with preserved ejection fraction, echocardiogram, circulating biomarkers, prognosis

\section{INTRODUCTION}

Rheumatoid arthritis (RA) is a chronic condition characterized by systemic inflammation affecting nearly $1 \%$ of the population (1). As a consequence of the underlying proinflammatory and profibrotic state, patients with RA have a high risk of progressing toward cardiac structural and functional abnormalities [e.g., left ventricular hypertrophy (LVH), left atrial enlargement, and diastolic dysfunction] (2, 3). These cardiac alterations may increase the risk of developing heart failure (HF) (4).

Circulating biomarkers may provide relevant information about the underlying pathophysiological processes associated with both the RA and cardiac alterations, even when asymptomatic $(5,6)$. Determining the circulating biomarkers that correlate with the cardiac structure and function of patients with RA may help to better identify individuals at a risk of developing symptomatic HF and implement biomarker-based screening strategies. In this regard, consistent associations between inflammatory biomarkers and abnormal diastolic function were reported in $\mathrm{HF}$ with preserved ejection fraction (HFpEF) likely because HFpEF is a condition characterized by high expression of inflammatory markers $(4,7)$.

The RA Porto cohort (ClinicalTrials.gov Identifier: NCT03960515) provides a good opportunity to explore the circulating biomarkers associated with echocardiographic parameters in RA and the prognostic value of echocardiographic parameters (8). Additionally, the associations between biomarkers and echocardiographic parameters were also assessed in a cohort of patients with HFpEF of the Metabolic Road to Diastolic Heart Failure (MEDIA-DHF) cohort study (ClinicalTrials.gov identifier: NCT02446327) (9) and a cohort of an initially healthy population in the Suivi Temporaire Annuel Non-Invasif de la Santé des Lorrains Assurés Sociaux (STANISLAS) (ClinicalTrials.gov identifier: NCTO1391442) (10).

\section{METHODS}

\section{Study Population}

This study is reported as per the Strengthening the Reporting of Observational Studies in Epidemiology (STROBE) guideline (Supplementary Table 1). The RA Porto cohort included 408 patients aged $\geq 18$ years with RA diagnosis according to the 2010 American College of Rheumatology/European League Against Rheumatism (ACR/EULAR) criteria (11) followed in the Autoimmune Disease Unit of Centro Hospitalar Universitário do Porto, Portugal from June 2016 to June 2018 as previously published (8). Demographic parameters, medical history, physical examination, laboratory findings and treatments, and RA-related information (i.e., RA duration period) were collected. Patient median follow-up time was 1,459 days ( 4.0 years). Cause of death and hospitalization were independently adjudicated. For the current analysis, we included patients with available data on echocardiogram and biomarkers. This study was conducted in accordance with the Declaration of Helsinki and approved by the Institutional Ethics Committees and all the participants signed a written informed consent prior to entry into this study.

The MEDIA-DHF cohort study included 626 patients with HFpEF diagnosis according to the 2007 European Society of Cardiology (ESC) consensus recommendations between 2012 and 2014 as previously published (9). A total of 392 patients with HFpEF with available data on echocardiographic parameters and biomarkers were included.

The STANISLAS cohort study is a single-center familial longitudinal population-based cohort from the Nancy region in France as previously reported (10). Among 1,705 participants undergoing the 4th cohort visit, 1,679 participants had available both the echocardiographic and biomarker data.

The data used for this report can be assessed by other authors upon reasonable request to the corresponding author.

\section{Echocardiography}

Echocardiographic parameters were acquired by one cardiologist that was blinded to clinical data and circulating biomarker values of patient by using the Philips ${ }^{\circledR}$ iE33 Ultrasound Machine, Philips, Bothell, WA. Cardiac chamber and systolic function [i.e., left ventricular ejection fraction (LVEF), left ventricular mass index (LVMi), left atrial volume index (LAVi), and inferior vena cava (IVC) diameter] were assessed according to the international recommendations $(12,13)$. Pulmonary arterial systolic pressure (PASP) was estimated by using peak tricuspid regurgitation (TR) velocity and IVC compliance and diameter (13). Diastolic function was assessed from the mitral inflow pattern by pulsedwave Doppler. Mitral annular early diastolic velocity (e') was assessed at the septal and lateral sites of the mitral annulus by using tissue Doppler imaging. E/A ratio, e' mean, and E/e' mean ratio were calculated (14). LVH was defined by a LVMi > 115 $\mathrm{g} / \mathrm{m}^{2}$ in males or $>95 \mathrm{~g} / \mathrm{m}^{2}$ in females (12). The measurement reproducibility is shown in Supplementary Table 2.

\section{Circulating Proteomic Biomarkers}

A large biomarker panel with 92 biomarkers from a wide range of pathophysiological domains was measured (Olink ${ }^{\circledR}$ CVDII panel). An overview of biomarkers, their full names, 
TABLE 1 | Baseline characteristics in the RA Porto cohort $(N=355)$.

\begin{tabular}{|c|c|}
\hline & Mean \pm SD or $N(\%)$ \\
\hline Age, yrs & $58.4 \pm 13.1$ \\
\hline Male, $N(\%)$ & $84(23.1 \%)$ \\
\hline Body mass index, $\mathrm{kg} / \mathrm{m}^{2}$ & $26.6 \pm 4.5$ \\
\hline \multicolumn{2}{|l|}{ Medical history, $N(\%)$} \\
\hline Hypertension & $170(46.8 \%)$ \\
\hline Diabetes & $51(14.0 \%)$ \\
\hline Dyslipidemia & $174(47.9 \%)$ \\
\hline Coronary artery disease & $8(2.2 \%)$ \\
\hline Heart failure & $115(32.4 \%)$ \\
\hline Atrial fibrillation & $13(3.6 \%)$ \\
\hline Chronic obstructive pulmonary disease & $18(5.0 \%)$ \\
\hline Smoking & $51(14.0 \%)$ \\
\hline NYHA $>$ III & $28(7.7 \%)$ \\
\hline Systolic blood pressure, bpm & $132.9 \pm 19.0$ \\
\hline Heart rate, bpm & $80.6 \pm 14.6$ \\
\hline \multicolumn{2}{|l|}{ RA history } \\
\hline RA diagnostic (years) & $11.5 \pm 10.1$ \\
\hline RF or anti CCP positive, $N(\%)$ & $277(78.0 \%)$ \\
\hline Articular erosions, $N(\%)$ & $123(34.6 \%)$ \\
\hline DAS28 VS (ESR) & $2.8 \pm 1.2$ \\
\hline DAS28 VS (CRP) & $2.4 \pm 1.1$ \\
\hline \multicolumn{2}{|l|}{ Medication } \\
\hline ACE inhibitor or ARB, $N(\%)$ & $136(38.3 \%)$ \\
\hline Beta-blocker, $N(\%)$ & $40(11.3 \%)$ \\
\hline Calcium channel blocker, N (\%) & $40(11.3 \%)$ \\
\hline Aldosterone antagonist, $N(\%)$ & $2(0.6 \%)$ \\
\hline Loop diuretics, $N(\%)$ & $10(2.8 \%)$ \\
\hline Statin, $N(\%)$ & $134(37.7 \%)$ \\
\hline Corticosteroids, N (\%) & $162(45.6 \%)$ \\
\hline Methotrexate, $N(\%)$ & $215(60.7 \%)$ \\
\hline NSAIDs, $N(\%)$ & $85(23.9 \%)$ \\
\hline Biological DMARDs, N (\%) & $65(18.3 \%)$ \\
\hline \multicolumn{2}{|l|}{ Individual DMNARDs } \\
\hline Anti-TNF $\alpha$ & $38(60.3 \%)$ \\
\hline Rituximab & $11(16.2 \%)$ \\
\hline Tocilizumab & $16(23.5 \%)$ \\
\hline \multicolumn{2}{|l|}{ Biochemistry } \\
\hline $\mathrm{HbA} 1 \mathrm{c}, \%$ & $5.6 \pm 0.8$ \\
\hline LDL cholesterol, mg/dl & $101.0 \pm 31.0$ \\
\hline Hemoglobin, g/dl & $13.1 \pm 1.4$ \\
\hline eGFR, $\mathrm{ml} / \mathrm{min} / 1.73 \mathrm{~m}^{2}$ & $88.3 \pm 20.3$ \\
\hline \multicolumn{2}{|l|}{ Echocardiogram } \\
\hline LVEF, \% & $61.0 \pm 7.1$ \\
\hline LVEF <50\%, N (\%) & $17(5.0 \%)$ \\
\hline LVMi, $g / m^{2}$ & $69.6 \pm 21.4$ \\
\hline LV hypertrophy, N (\%) & $32(9.0 \%)$ \\
\hline LAVi, $\mathrm{ml} / \mathrm{m}^{2}$ & $32.4 \pm 10.9$ \\
\hline LAVi > 34 ml/m², N (\%) & $129(35.7 \%)$ \\
\hline E/A ratio & $1.0 \pm 0.7$ \\
\hline$e^{\prime}$ mean, $\mathrm{cm} / \mathrm{s}$ & $8.9 \pm 2.7$ \\
\hline Septal $\mathrm{e}^{\prime}<8 \mathrm{~cm} / \mathrm{s}$ or I ateral $\mathrm{e}^{\prime}<10 \mathrm{~cm} / \mathrm{s}, N(\%)$ & $205(57.7 \%)$ \\
\hline E/e' mean & $9.3 \pm 3.9$ \\
\hline
\end{tabular}

(Continued)
TABLE 1 | Continued

\begin{tabular}{lc}
\hline & Mean \pm SD or $\boldsymbol{N}(\%)$ \\
\hline E/e' mean $>14, N(\%)$ & $30(8.4 \%)$ \\
Peak TR velocity, $\mathrm{cm} / \mathrm{s}(N=178)$ & $2.3 \pm 0.4$ \\
Peak TR velocity $>2.8 \mathrm{~cm} / \mathrm{s}, N(\%)(N=178)$ & $12(6.7 \%)$ \\
Pulmonary artery systolic pressure, $\mathrm{mmHg}$ & $23.7 \pm 7.6$ \\
\hline
\end{tabular}

Values are mean $\pm S D, n(\%)$ or median (25th-75th percentile).

NYHA, New York Heart Association; RA, rheumatoid arthritis; anti-CCP, anti-citrulline antibody; DAS28 VS (ESR), Disease Activity Score for Rheumatoid Arthritis with Erythrocyte Sedimentation Rate; ACE inhibitor, angiotensin converting enzyme inhibitor; $A R B$, angiotensin receptor blocker; NSAIDs, nonsteroidal anti-inflammatory drugs; DMARDs, disease-modifying anti-rheumatic drugs; HbA1c, glycosylated hemoglobin; $L D L$, low-density lipoprotein; eGFR, estimated glomerular filtration rate; LVEF, left ventricular ejection fraction; LVMi, left ventricular mass index; LAVi, left atrial volume index; $T R$, tricuspid regurgitation.

UniProt ID, and roles are presented in Supplementary Table 3. These biomarkers were measured by using a high-throughput technique by using the Olink Proseek ${ }^{\circledR}$ Multiplex CVDII $96 \times$ 96 kit, which measures 92 biomarkers simultaneously in $1-\mu 1$ plasma samples. The kit uses a proximity extension assay (PEA) technology where 92 oligonucleotide-labeled antibody probe pairs allowed to bind to their respective target present in the sample. Biomarker expression is provided in a Log2-normalized scale, Normalized Protein eXpression (NPX). For more details, please go to http://www.olink.com/ (15). In addition to the Olink ${ }^{\circledR}$ CVDII panel, in the RA Porto cohort, N-terminal pro-brain natriuretic peptide (NT-proBNP) and high-sensitivity troponin $\mathrm{T}$ (hsTnT) were measured by the Elecsys (Roche Diagnostics ${ }^{\circledR}, G m b H$, Penzberg, Germany) NT-proBNP and troponin $\mathrm{T}$ (Gen 5 STAT test). In the MEDIA-DHF and the STANISLAS cohorts, NT-proBNP and high-sensitivity troponin I (hsTnI) were, respectively, measured by the Olink ${ }^{\circledR}$ CVDIII and organ damage panel.

\section{Statistical Analysis}

Categorical variables are described as frequencies (percentages), while continuous variables are described as mean \pm SD or median (25th and 75th percentiles) depending on the variable distributions. The multivariable linear regression analysis was performed to test the associations between the circulating biomarkers and echocardiographic parameters. Models included relevant confounders as previously shown $(3,16)$ : age, sex, body mass index (BMI), diabetes, systolic blood pressure, estimated glomerular filtration rate (eGFR) [the Chronic Kidney Disease Epidemiology Collaboration formula (17)], and RA duration. In the MEDIA-DHF and the STANISLAS cohorts, the above confounders excluding RA duration were adjusted to assess the associations between biomarkers and echocardiographic parameters. Multiple testing correction for false discoveries was set at 5\% (false discover rate $(\mathrm{FDR})<0.05)$, as described by Benjamini and Hochberg (18).

In the RA Porto cohort, the primary outcome was the composite of cardiovascular mortality or hospitalization for cardiovascular reasons. Hospitalization for cardiovascular 
TABLE 2 | Multivariable selected biomarkers for each echocardiographic parameter in the RA porto cohort.

\begin{tabular}{|c|c|c|c|c|c|c|c|c|c|c|c|}
\hline \multicolumn{4}{|c|}{ LVMi (per 1 g/m²) } & \multicolumn{4}{|c|}{ LAVi (per $1 \mathrm{ml} / \mathrm{m}^{2}$ ) } & \multicolumn{4}{|c|}{$E / e^{\prime}$ mean (per 1) } \\
\hline BMP9 & $-6.30(-9.88$ to -2.72$)$ & $<0.001$ & 0.01 & IL4RA & 5.71 (2.51 to 8.91$)$ & $<0.001$ & 0.01 & PGF & $2.63(1.56$ to 3.7$)$ & $<0.001$ & $<0.001$ \\
\hline Troponin & 7.25 (3.87 to 10.62$)$ & $<0.001$ & $<0.001$ & THBS2 & 9.35 (4.39 to 14.31$)$ & $<0.001$ & 0.01 & TNFRSF11A & 1.09 (0.31 to 1.88$)$ & 0.006 & 0.046 \\
\hline BNP & 4.12 (2.36 to 5.88$)$ & $<0.001$ & $<0.001$ & AGRP & 5.22 (2.7 to 7.74$)$ & $<0.001$ & $<0.001$ & TIE2 & 2.17 (0.61 to 3.72 ) & 0.006 & 0.046 \\
\hline & & & & $\mathrm{BNP}$ & 3.79 (2.90 to 4.68$)$ & $<0.001$ & $<0.001$ & THBS2 & 2.42 (0.69 to 4.14$)$ & 0.006 & 0.046 \\
\hline & & & & NTproBNP & 2.90 (1.84 to 3.96$)$ & $<0.001$ & $<0.001$ & PRELP & 2.78 (0.90 to 4.66$)$ & 0.004 & 0.045 \\
\hline & & & & & & & & PTX3 & 1.14 (0.45 to 1.84$)$ & 0.001 & 0.02 \\
\hline & & & & & & & & CTSL1 & 1.19 (0.35 to 2.03$)$ & 0.006 & 0.046 \\
\hline & & & & & & & & CD4 & 1.76 (0.68 to 2.83 ) & 0.001 & 0.02 \\
\hline & & & & & & & & Troponin & 0.95 (0.32 to 1.58$)$ & 0.003 & 0.04 \\
\hline
\end{tabular}

Multivariable model was adjusted for age, sex, body mass index, diabetes, systolic blood pressure, estimated glomerular filtration rate and rheumatic arthritis disease duration. KIM1, Kidney injury molecule 1; BMP9, Bone morphogenetic protein 9; BNP, B-type natriuretic peptide; NTproBNP, N-terminal pro b-type natriuretic peptide; SLAMF7, SLAM family member 7; IL4RA, Interleukin-4 receptor subunit alpha; THBS2, Thrombospondin-2; AGRP, Agouti-related protein; CD4, T-cell surface glycoprotein CD4; ADM, Adrenomedullin; PGF, Placenta growth factor; TNFRSF11A, Tumor necrosis factor receptor superfamily member 11A; TIE2, Angiopoietin-1 receptor; SPON2, Spondin-2; PRELP, Prolargin; PTX3, Pentraxin-related protein; CTSL1, Cathepsin L1.

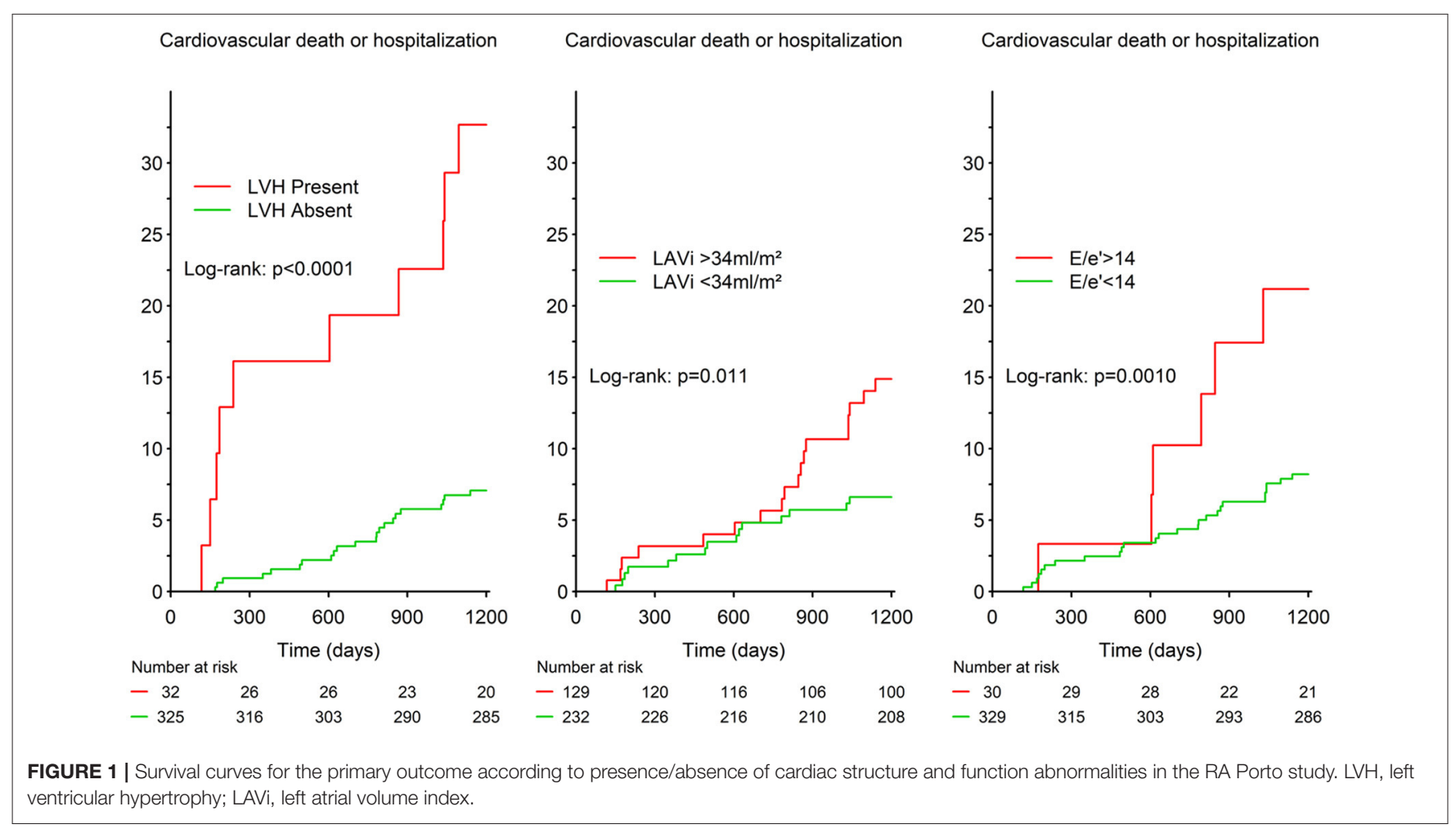

events included HF hospitalization, myocardial infarction, acute coronary syndromes, angina pectoris, stroke, transient ischemic attack, and peripheral artery diseases. Survival probabilities were estimated by using the KaplanMeier method. The Cox proportional hazards model for echocardiographic structure/function abnormalities was used to obtain the unadjusted and covariate adjusted hazard ratios. In addition, we explored the associations of biomarkers with the outcome after adjustment for echocardiographic parameters plus clinical covariates. No data imputation was performed.

All the statistical analyses were performed by using the $\mathrm{R}$ software version 4.0.1 ( $\mathrm{R}$ Development Core Team, Vienna, Austria, UK). 


\section{RESULTS}

\section{Baseline Characteristics}

The mean age of the 355 patients with RA included in the RA Porto cohort was $58 \pm 13$ years, 23\% were males, $20 \%$ were obese $\left(B M I \geq 30 \mathrm{~kg} / \mathrm{m}^{2}\right)$, and $47 \%$ had hypertension. The mean RA duration of these patients was $12 \pm 10$ years, $34.6 \%$ had an erosive disease, and $45.6 \%$ took corticosteroids. The mean LVEF was $61 \pm 7 \%$, 9.0\% had LVH based on LVMi, 36\% had left atrial enlargement $\left(\mathrm{LAVi} \geq 34 \mathrm{ml} / \mathrm{m}^{2}\right)$, and $8.4 \%$ had elevated $\mathrm{E} / \mathrm{e}^{\prime}$ ratio $\left(\mathrm{E} / \mathrm{e}^{\prime}>14\right)$ (Table 1).

\section{Association of Circulating Biomarkers With Echocardiographic Parameters}

The circulating biomarkers associated with echocardiographic parameters are shown in the Supplementary Tables 4-6.

In patients with RA, LVMi was positively associated with higher levels of hsTnT and BNP/NT-proBNP and negatively associated with bone morphogenetic protein 9 (BMP9); LAVi was positively associated with BNP/NT-proBNP, interleukin-4 receptor subunit alpha 4 (IL4RA4), T-cell surface glycoprotein cluster of differentiation 4 (CD4), and thrombospondin 2 (THBS2); E/e' was positively associated with hsTnT, BNP/NTproBNP, adrenomedullin (ADM), placental growth factor (PGF), spondin-2 (SPON-2), THBS2, tumor necrosis factor receptor superfamily member 11a (TNFRSF11A), pentraxinrelated protein 3 (PTX3), cathepsin L1 (CTSL1), and CD4 (FDR, $q<0.001$ ) (Table 2).

In the multivariable logistic regression analysis, higher levels of BNP/NT-proBNP were associated with cardiac structural and functional abnormalities (i.e., LVH, LAVi > $34 \mathrm{ml} / \mathrm{m}^{2}$, E/e' $>14$ ) and higher PGF level was associated with $\mathrm{E} / \mathrm{e}^{\prime}>14$ (Supplementary Table 7).

Similar associations were found in population of patients with HFpEF and asymptomatic individuals (Supplementary Table 8).

\section{Echocardiographic Parameters, Circulating Biomarkers, and Clinical Outcomes}

In patients with RA during a median follow-up of 48 months, the primary outcome $(n=37,10.2 \%)$ occurred more frequently in patients with abnormalities of cardiac structure and function (Figure 1). In univariable model, greater LVMi [hazard ratio (HR) (95\% CI) per $\left.1 \mathrm{~g} / \mathrm{m}^{2}=1.03(1.02-1.04), p<0.001\right]$, greater LAVi [HR $(95 \% \mathrm{CI})$ per $1 \mathrm{ml} / \mathrm{m}^{2}=1.03(1.01-1.06), p$ $<0.001$ ], and higher E/e' $[\mathrm{HR}(95 \% \mathrm{CI})$ per $1=1.08(1.04-1.13)$, $p<0.001]$ were associated with higher rates of cardiovascular events. After adjustment for potential confounders, the poor prognosis associated with greater LVMi and higher E/e' persisted $(p<0.05$; Table 3).

Furthermore, higher levels of TNF-related apoptosisinducing ligand receptor 2 (TRAILR2) and CTSL1 were significantly associated with higher risk of the outcome after adjustment for echocardiographic parameters plus clinical confounders (Table 4).
TABLE 3 | Survival analyses for the primary outcome in the RA Porto cohort.

\begin{tabular}{|c|c|c|c|c|}
\hline & \multicolumn{2}{|c|}{ Univariable model } & \multicolumn{2}{|c|}{ Multivariable model } \\
\hline & HR $(95 \% \mathrm{Cl})$ & $p$-value & HR $(95 \% \mathrm{Cl})$ & $p$-value \\
\hline LVMi (per $\left.1 \mathrm{~g} / \mathrm{m}^{2}\right)$ & $\begin{array}{c}1.03 \\
(1.02-1.04)\end{array}$ & $<0.001$ & $\begin{array}{c}1.03 \\
(1.01-1.04)\end{array}$ & $<0.001$ \\
\hline $\mathrm{LVH}$ & $\begin{array}{c}5.38 \\
(2.65-10.94)\end{array}$ & $<0.001$ & $\begin{array}{c}4.55 \\
(2.17-9.51)\end{array}$ & $<0.001$ \\
\hline LAVi (per $1 \mathrm{ml} / \mathrm{m}^{2}$ ) & $\begin{array}{c}1.03 \\
(1.01-1.06)\end{array}$ & 0.004 & $\begin{array}{c}1.02 \\
(0.99-1.04)\end{array}$ & 0.21 \\
\hline $\mathrm{LAVi}>34 \mathrm{ml} / \mathrm{m}^{2}$ & $\begin{array}{c}2.27 \\
(1.19-4.33)\end{array}$ & 0.01 & $\begin{array}{c}1.71 \\
(0.88-3.32)\end{array}$ & 0.12 \\
\hline $\mathrm{E} / \mathrm{e}^{\prime}($ per 1$)$ & $\begin{array}{c}1.08 \\
(1.04-1.13)\end{array}$ & $<0.001$ & $\begin{array}{c}1.06 \\
(1.002-1.13)\end{array}$ & 0.042 \\
\hline$E / e^{\prime}$ mean $>14$ & $\begin{array}{c}3.47 \\
(1.58-7.61)\end{array}$ & 0.002 & $\begin{array}{c}2.44 \\
(1.03-5.77)\end{array}$ & 0.042 \\
\hline
\end{tabular}

Multivariable model included age, sex, body mass index, diabetes, systolic blood pressure, estimated glomerular filtration rate and rheumatoid arthritis duration. LVMi, left ventricular mass indexed; LVH, left ventricular hypertrophy; LAVi, left atrial volume indexed.

\section{DISCUSSION}

This study shows that cardiac structure (i.e., LVMi and LAVi) and diastolic function (i.e., E/e') of patients with RA were associated with circulating biomarkers reflecting inflammation (i.e., BMP9, PTX3, and TNFRSF11A), adverse extracellular matrix remodeling (i.e., PGF), congestion (i.e., NT-proBNP and $\mathrm{ADM}$ ), and myocardial injury (i.e., hsTnT). Alterations of these echocardiographic parameters were associated with a worse prognosis. In addition, higher levels of inflammatory biomarkers (i.e., TRAILR2 and CTSL1) were associated with poorer prognosis in RA, independent of these cardiac alterations. These associations were also found in a population of patients with HFpEF and asymptomatic individuals. Together, these findings suggest that biomarker-guided screening may be relevant to detect cardiac structure and functional alterations in patients with RA, who are at a high risk of cardiovascular events.

The inflammatory biomarker associations described here make biologic and pathophysiological sense. We observed that a greater LVMi was associated with lower BMP9 levels and higher NT-proBNP and hsTnT levels. BMP9, a member of transforming growth factor- $\beta 1$, as a potent anti-inflammatory cytokine, is expressed in the blood and heart tissues in HF. This protein inhibits cardiac fibrosis and reduces LV mass in an experimental model of HF (19). In addition, NT-proBNP and hsTnT are strongly associated with abnormalities of left ventricle and with cardiovascular morbidity and/or mortality even in pre-clinical settings $(20,21)$.

E/e' reflects left ventricular filling pressures and carries relevant prognostic value $(22,23)$. Several biomarkers reflecting systemic inflammation (e.g., TNFRSF11A, PTX3, CTSL1, and CD4) were increased in patients with RA, which may reflect a link between RA-associated inflammation and diastolic dysfunction (7). Other biomarkers associated with higher $\mathrm{E} / \mathrm{e}^{\prime}$ included BNP/NT-proBNP, hsTnT, ADM, PGF, SPON-2, and THBS2. As $\mathrm{E} / \mathrm{e}^{\prime}$ reflects left ventricular filling pressures and diastolic 
stiffness, it is not surprising its association with these biomarkers. For example, ADM is a vasoactive peptide synthesized by endothelial and vascular smooth muscle cells, which is increased by endothelial dysfunction and volume overload (24). PGF, a member of the vascular endothelial growth factor family, is expressed in myocytes and is increased by pressure and volume overload (25). SPON-2 expression has been associated with inflammatory processes and cardiac fibrosis (26). THBS2 has been associated with the severity of congestion in HFpEF (27). Concordantly, LAVi, reflecting left ventricular filling pressure, was also associated with many inflammatory biomarkers (e.g., CD4 and IL4RA).

Interestingly, TRAILR2 and CTSL1 expressed inflammation and apoptosis and were related to inflammatory status in RA $(28,29)$. Therefore, our observations showing higher levels of these biomarkers were associated with a higher incident outcome that supported an adverse prognosis of enhanced levels of inflammatory biomarkers in patients with RA.

Several algorithms combining LVMi, LAVi, and E/e' have been proposed for diagnosing or grading diastolic function $(14,30)$. Our findings suggest that inflammatory biomarkers and widely available biomarkers as BNP/NT-proBNP and hsTnT should be used for the screening of cardiac alterations in patients with RA and asymptomatic individuals. These biomarkers may be used for an early detection of asymptomatic cardiac dysfunction and prompt an adequate control of cardiovascular and RA-related risk factors (e.g., hypertension, diabetes, and RA activity).

\section{LIMITATIONS}

This study had several limitations. First, all the three cohorts are observational cohort studies; thus, causality cannot be inferred. Second, some patients in the RA Porto cohort did not undergo echocardiogram and were excluded from the final analysis. Third, the RA Porto and the STANISLAS cohorts came from a single center and some of the findings may be influenced by local echocardiographers or practice patterns. Fourth, abnormalities of cardiac structure and function were reported to be independent of traditional cardiac risk factors and coronary artery disease in patients with RA $(31,32)$; however, cardiovascular comorbidities (i.e., coronary artery disease and HF) may influence the associations between echocardiographic parameters and biomarkers. We observed consistent associations between biomarkers and echocardiographic parameters across the different populations (i.e., patients with RA, those with HFpEF, and asymptomatic individuals) and biomarkers associated with study outcomes. However, external validation in other cohorts of patients with RA is needed.

\section{CONCLUSION}

Circulating biomarkers reflecting inflammation, extracellular matrix inflammation, remodeling, congestion, and myocardial injury were associated with underlying alterations of cardiac structure and function. Biomarkers might be used for the screening of cardiac alterations in patients with RA. 


\section{DATA AVAILABILITY STATEMENT}

The raw data supporting the conclusions of this article will be made available by the authors, without undue reservation.

\section{ETHICS STATEMENT}

The studies involving human participants were reviewed and approved by Centro Hospitalar do Porto with the number 2016-023 (020-DEFI/020-CES). The patients/participants provided their written informed consent to participate in this study.

\section{REFERENCES}

1. Gabriel SE, Crowson CS, Kremers HM, Doran MF, Turesson C, O'Fallon WM, et al. Survival in rheumatoid arthritis: a population-based analysis of trends over 40 years. Arthritis Rheum. (2003) 48:54-8. doi: 10.1002/art.10705

2. Mason JC, Libby P, Cardiovascular disease in patients with chronic inflammation: mechanisms underlying premature cardiovascular events in rheumatologic conditions. Eur Heart J. (2015) 36:482-9c. doi: 10.1093/eurheartj/ehu403

3. Liang KP, Myasoedova E, Crowson CS, Davis JM, Roger VL, Karon BL, et al. Increased prevalence of diastolic dysfunction in rheumatoid arthritis. Ann Rheum Dis. (2010) 69:1665-70. doi: 10.1136/ard.2009.124362

4. Packer M, Lam CSP, Lund LH, Maurer MS, Borlaug BA. Characterization of the inflammatory-metabolic phenotype of heart failure with a preserved ejection fraction: a hypothesis to explain influence of sex on the evolution and potential treatment of the disease. Eur J Heart Fail. (2020) 22:1551-67. doi: 10.1002/ejhf.1902

5. Cesari M, Penninx BW, Newman AB, Kritchevsky SB, Nicklas BJ, SuttonTyrrell $\mathrm{K}$, et al. Inflammatory markers and onset of cardiovascular events: results from the Health ABC study. Circulation. (2003) 108:2317-22. doi: 10.1161/01.CIR.0000097109.90783.FC

6. Zile MR, Desantis SM, Baicu CF, Stroud RE, Thompson SB, McClure CD, et al. Plasma biomarkers that reflect determinants of matrix composition identify the presence of left ventricular hypertrophy and diastolic heart failure. Circ Heart Fail. (2011) 4:246-56. doi: 10.1161/CIRCHEARTFAILURE.110.958199

7. Paulus WJ, Tschöpe C. A novel paradigm for heart failure with preserved ejection fraction: comorbidities drive myocardial dysfunction and remodeling through coronary microvascular endothelial inflammation. J Am Coll Cardiol. (2013) 62:263-71. doi: 10.1016/j.jacc.2013.02.092

8. Ferreira MB, Fonseca T, Costa R, Marinhoc A, Carvalho HC, Oliveira JC, et al. Prevalence, risk factors and proteomic bioprofiles associated with heart failure in rheumatoid arthritis: the RA-HF study. Eur J Intern Med. (2021) 85:41-9. doi: 10.1016/j.ejim.2020.11.002

9. Stienen S, Ferreira JP, Kobayashi M, Preud'homme G, Dobre D, Machu JL, et al. Enhanced clinical phenotyping by mechanistic bioprofiling in heart failure with preserved ejection fraction: insights from the MEDIA-DHF study (The Metabolic Road to Diastolic Heart Failure). Biomarkers. (2020) 25:20111. doi: 10.1080/1354750X.2020.1727015

10. Ferreira JP, Girerd N, Bozec E, Merckle L, Pizard A, Bouali S, et al. Cohort profile: rationale and design of the fourth visit of the STANISLAS cohort: a familial longitudinal population-based cohort from the Nancy region of France. Int J Epidemiol. (2018) 47:395. doi: 10.1093/ije/dyx240

11. Villeneuve ENJ, Emery P, (2010). ACR-EULAR classification criteria for rheumatoid arthritis. Rev Bras Reumatol. (2010) 50:481-3. doi: 10.1590/S0482-50042010000500001

12. Lang RM BL, Mor-Avi V, Afilalo J, Armstrong A, Ernande L, Flachskampf FA, et al. Recommendations for cardiac chamber quantification by echocardiography in adults: an update from the American Society of Echocardiography and the European Association of Cardiovascular Imaging. Eur Heart J Cardiovasc Imaging. (2015) 16:233-70. doi: 10.1093/ehjci/jev014

\section{AUTHOR CONTRIBUTIONS}

MK and MF: drafting. JF: supervision. JF, PRod, NG, PRos, and FZ: critical review of the manuscript. MF, RC, TF, JO, AM, and HC: data collection. MK and JF: analysis. All authors contributed to the article and approved the submitted version.

\section{SUPPLEMENTARY MATERIAL}

The Supplementary Material for this article can be found online at: https://www.frontiersin.org/articles/10.3389/fcvm. 2021.754784/full\#supplementary-material

13. Rudski LG, Lai WW, Afilalo J, Hua L, Handschumacher MD, Chandrasekaran $\mathrm{K}$, et al. Guidelines for the echocardiographic assessment of the right heart in adults: a report from the American Society of Echocardiography endorsed by the European Association of Echocardiography, a registered branch of the European Society of Cardiology, and the Canadian Society of Echocardiography. J Am Soc Echocardiogr. (2010) 23:685-713; quiz 786-8. doi: 10.1016/j.echo.2010.05.010

14. Nagueh SF, Smiseth OA, Appleton CP, Byrd BF, III, Dokainish H, Edvardsen $\mathrm{T}$, et al. Recommendations for the evaluation of left ventricular diastolic function by echocardiography: an update from the American Society of Echocardiography and the European Association of Cardiovascular Imaging. J Am Soc Echocardiogr. (2016) 29:277-314. doi: 10.1016/j.echo.2016.01. 011

15. Enroth S, Maturi V, Berggrund M, Enroth SB, Moustakas A, Johansson A, et al. Systemic and specific effects of antihypertensive and lipid-lowering medication on plasma protein biomarkers for cardiovascular diseases. Sci Rep. (2018) 8:5531. doi: 10.1038/s41598-018-23860-y

16. Yavasoglu I, Senturk T, Onbasili A. Diastolic dysfunction in rheumatoid arthritis and duration of disease. Rheumatol Int. (2008) 29:113-4. doi: 10.1007/s00296-008-0625-5

17. Levey AS, Stevens LA, Schmid CH, Zhang YL, Castro AF, III, Feldman HI, et al. A new equation to estimate glomerular filtration rate. Ann Intern Med. (2009) 150:604-12. doi: 10.7326/0003-4819-150-9-200905050-00006

18. Boccard J, Rutledge DN. A consensus orthogonal partial least squares discriminant analysis (OPLS-DA) strategy for multiblock Omics data fusion. Anal Chim Acta. (2013) 769:30-9. doi: 10.1016/j.aca.2013.01.022

19. Morine KJ, Qiao X, York S, Natov PS, Paruchuri V, Zhang Y, et al. Bone morphogenetic protein 9 reduces cardiac fibrosis and improves cardiac function in heart failure. Circulation. (2018) 138:513-26. doi: 10.1161/CIRCULATIONAHA.117.031635

20. Lukowicz TV, Fischer M, Hense HW, Döring A, Stritzke J, Riegger G, et al. BNP as a marker of diastolic dysfunction in the general population: importance of left ventricular hypertrophy. Eur J Heart Fail. (2005) 7:525-31. doi: 10.1016/j.ejheart.2004.12.010

21. de Lemos JA, Drazner MH, Omland T, Ayers CR, Khera A, Rohatgi A, et al. Association of troponin $\mathrm{T}$ detected with a highly sensitive assay and cardiac structure and mortality risk in the general population. JAMA. (2010) 304:2503-12. doi: 10.1001/jama.2010.1768

22. Shah AM, Cikes M, Prasad N, Li G, Getchevski S, Claggett B, et al. Echocardiographic features of patients with heart failure and preserved left ventricular ejection fraction. J Am Coll Cardiol. (2019) 74:2858-73. doi: 10.1016/j.jacc.2019.09.063

23. Nagueh SF. Left ventricular diastolic function: understanding pathophysiology, diagnosis, and prognosis with echocardiography. JACC Cardiovasc Imaging. (2019) 13:228-44. doi: 10.1016/j.jcmg.2018.10.038

24. Voors AA, Kremer D, Geven C, Ter Maaten JM, Struck J, Bergmann A, et al. Adrenomedullin in heart failure: pathophysiology and therapeutic application. Eur J Heart Fail. (2019) 21:163-71. doi: 10.1002/ejhf.1366

25. Ky B, French B, Ruparel K, Sweitzer NK, Fang JC, Levy WC, et al. The vascular marker soluble fms-like tyrosine kinase 1 is associated with disease severity 
and adverse outcomes in chronic heart failure. J Am Coll Cardiol. (2011) 58:386-94. doi: 10.1016/j.jacc.2011.03.032

26. Dogan I, Yetim M, Dogan T, Kayadibi H, Yilmaz MB, Eser B, et al. Relation of serum spondin-2 levels with cardiac morphology and inflammatory parameters in hemodialysis patients. Int Urol Nephrol. (2018) 50:2091-7. doi: 10.1007/s11255-018-1996-5

27. Kimura $Y$, Izumiya $Y$, Hanatani S, Yamamoto E, Kusaka H, Tokitsu T, e al. High serum levels of thrombospondin-2 correlate with poor prognosis of patients with heart failure with preserved ejection fraction. Heart Vessels. (2016) 31:52-9. doi: 10.1007/s00380-014-0571-y

28. Cunnane G, FitzGerald O, Hummel KM, Youssef PP, Gay RE, Gay $\mathrm{S}$, et al. Synovial tissue protease gene expression and joint erosions in early rheumatoid arthritis. Arth Rheum. (2001) 44:1744-53. doi: 10.1002/1529-0131(200108)44:8<1744::AID-ART309>3.0.CO2-K

29. Ichikawa K, Liu W, Fleck M, Zhang H, Zhao L, Ohtsuka T, et al. TRAILR2 (DR5) mediates apoptosis of synovial fibroblasts in rheumatoid arthritis. J Immunol. (2003) 171:1061-9. doi: 10.4049/jimmunol.171.2.1061

30. Paulus WJ, Tschope C, Sanderson JE, Rusconi C, Flachskampf FA, Rademakers FE, et al. How to diagnose diastolic heart failure: a consensus statement on the diagnosis of heart failure with normal left ventricular ejection fraction by the Heart Failure and Echocardiography Associations of the European Society of Cardiology. Eur Heart J. (2007) 28:2539-50. doi: 10.1093/eurheartj/ehm037

31. Davis JM, III, Lin G, Oh JK, Crowson CS, Achenbach SJ, Therneau TM, et al. Five-year changes in cardiac structure and function in patients with rheumatoid arthritis compared with the general population. Int J Cardiol. (2017) 240:379-85. doi: 10.1016/j.ijcard.2017.03.108

32. Mantel Ä, Holmqvist M, Andersson DC, Lund LH, Askling J. Association between rheumatoid arthritis and risk of ischemic and nonischemic heart failure. J Am Coll Cardiol. (2017) 69:1275-85. doi: 10.1016/j.jacc.2016.12.033

Conflict of Interest: The authors declare that the research was conducted in the absence of any commercial or financial relationships that could be construed as a potential conflict of interest.

Publisher's Note: All claims expressed in this article are solely those of the authors and do not necessarily represent those of their affiliated organizations, or those of the publisher, the editors and the reviewers. Any product that may be evaluated in this article, or claim that may be made by its manufacturer, is not guaranteed or endorsed by the publisher.

Copyright $\odot 2021$ Kobayashi, Ferreira, Costa, Fonseca, Oliveira, Marinho, Carvalho, Girerd, Rossignol, Zannad, Rodrigues and Ferreira. This is an open-access article distributed under the terms of the Creative Commons Attribution License (CC BY). The use, distribution or reproduction in other forums is permitted, provided the original author(s) and the copyright owner(s) are credited and that the original publication in this journal is cited, in accordance with accepted academic practice. No use, distribution or reproduction is permitted which does not comply with these terms. 